\title{
Driving collaborative improvement processes
}

\author{
Rick Middel*, José Gieskes*, Olaf Fisscher* \\ * Department of Technology \& Organisation, University of Twente, 7500 AE Enschede, \\ The Netherlands
}

\begin{abstract}
Continuous Improvement is a consolidated concept in theory and practice, mainly in the context of stand-alone companies. However, the battlefield of competition is increasingly moving from the level of individual firms to that of organisational settings based on loose company boundaries and collaborative relations among different units, such as the Extended Manufacturing Enterprises (EMEs). The concept of continuous improvement has hardly been applied in inter-organisational settings. The purpose of this paper is to propose preliminary theory on Collaborative Improvement (CoI), i.e. continuous improvement at the EME level. Based on a literature study on supply networks and continuous improvement, evidence from an in-depth case study of a large Dutch system integrator in the automotive industry and three of its suppliers, a model of CoI is proposed, explaining how collaborative improvement takes place within the EME context.
\end{abstract}

\section{Keywords: collaborative improvement, Extended Manufacturing Enterprise, continuous improvement}

\section{Introduction}

The battlefield of competition is moving from the level of the individual enterprises to that of Extended Manufacturing Enterprises (EME). In recent years, organisations had to cope with, amongst others, rapidly changing market demands, intensified international competition, rapidly changing technology developments and changing governmental restrictions (Porter 1990, Hamel and Prahalad 1989, Kanter 1994). In order to cope with these changes and stay competitive an organisation has to change/improve its performance continuously (Douma 1997). Continuous Improvement (CI) is a consolidated concept in managerial theory and practice and is considered vital in today's business environments, but is mainly dealt with in the context of stand-alone companies. As firms are forced to reexamine, at a strategic level, the way they do business in order to add value and reduce costs it becomes clear that the individual firm is an insufficient entity for identifying improvements (Harland et al. 1999). Therefore CI must be applied and used in interorganisational settings. However there is still a substantial lack of empirically grounded contributions to the concept and implementation of CI in an inter-organisational context. EMEs can hardly rely on traditional mechanisms supporting continuous improvement within firms, due to functional, geographical and time related barriers. That is why, EMEs need new approaches and tools to enable and enhance the business performance and collaboration between the partners involved.

This paper presents the results of an in-depth case study into the areas where continuous improvement in an EME context is and could be applied and the requirements of companies in terms of organisational, managerial and technological mechanisms to support and foster 
collaborative improvement. The case-study results were used to develop a model for the implementation of Collaborative Improvement (CoI). The in-depth case study and the development of a model are part of a three years EU-research project (Collaborative Improvement Tool for the Extended Manufacturing Enterprise, G1RD - CT2000 - 00299), a collaboration between different academic and industrial partners ${ }^{1}$. The overall purpose of the CO-IMPROVE project is to develop a tool for the implementation and support of CoI within the EME. In doing so, requirements of the companies with regard to the collaborative continuous improvement process should be identified and understood to design and model the collaborative improvement processes between the companies within the EME.

This paper is structured as follows. First, we will discuss the research background of this project, which presents a review of the literature in the fields of supply networks and continuous improvement related to the topic and scope described in this paper. Further we put a definition to the term Collaborative Improvement. Second, we discuss the research problem and the research questions and explain the research methodology. Next, we describe the in-depth case study in a Dutch EME and discuss its results in terms of requirements with regard to the implementation and sustainment of collaborative improvement in the Dutch EME. Based on the evidence of the case study a model of Collaborative Improvement is proposed of how to support and foster collaborative improvement activities within the EME. Finally, the last section reflects on and discusses the relevance of the research and highlights challenges for future research.

\section{Extended Manufacturing Enterprises and the collaborative improvement concept}

\section{Extended Manufacturing Enterprise}

Interfirm relationships have become increasingly fashionable during the last decades. Theories about networks of firms have developed since the early eighties. The idea is that the firm needs to look outside its boundaries to find all the resources and competencies needed to produce its products or deliver its services. The result is a restructuring of roles, responsibilities and organisational structures to align interfirm relationships with the market demands (Rich and Hines 1997). These relationships are fuelled, according to Douma (1997), by a number of global developments: internationalisation of markets, increasing complexity of technologies and increasing speed with which innovations take place. The need for developing these kind of relationships is widely discussed in the literature. A major stream is based on the transaction cost theory (Coase 1937, Williamson 1983, Dyer 1997), which considers collaboration as the form of relationship that minimises the total cost of the transaction.

The basic mechanism that characterises network relations is collaboration. Collaboration between companies consists of working together, over an extended period of time, for the benefit of both (Ring and Van de Ven 1992). Smith et al. (1991) define collaboration as a form of horizontal integration where companies operating in similar or related activities establish joint agreements for technology and information exchange. According to

\footnotetext{
${ }^{1}$ Members of the CO-IMPROVE consortium: Aalborg University (Denmark), Politecnico di Milano (Italy), Trinity College Dublin (Ireland), University of Twente (the Netherlands), Aermacchi (Italy), Power Packer Europe (the Netherlands), Sauer Danfoss (Denmark), IFS (Sweden), I2S (Greece)
} 
Spekman et al. (1998) collaboration is the last step of a transition from open-market negotiation to joint agreement relations. They see the relationships evolve from openmarket negotiation, through co-operation, co-ordination, to collaboration, which is characterised by supply chain integration, joint planning and technology sharing among partners. Collaboration brings about the idea of interdependence between actors, shared goals and vision, information and technology exchange, joint work and activities (Lamming 1993, Mohr and Spekman 1994). All these ideas are combined in enterprises, which extend each other in knowledge and capacities, leading to the concept of Extended Manufacturing Enterprises (Busby and Fan 1993, Stock 2000). An EME is a collection of strategically aligned dyadic relationships and the inter-dependencies between the dyads (see figure 1). The firms within the EME combine their activities, knowledge and capabilities on a structural, durable and joint basis in order to maximise the benefits for the involved companies. This means that they build channels between themselves through which information and knowledge can be exchanged, which allow the companies within the EME to act rapidly and

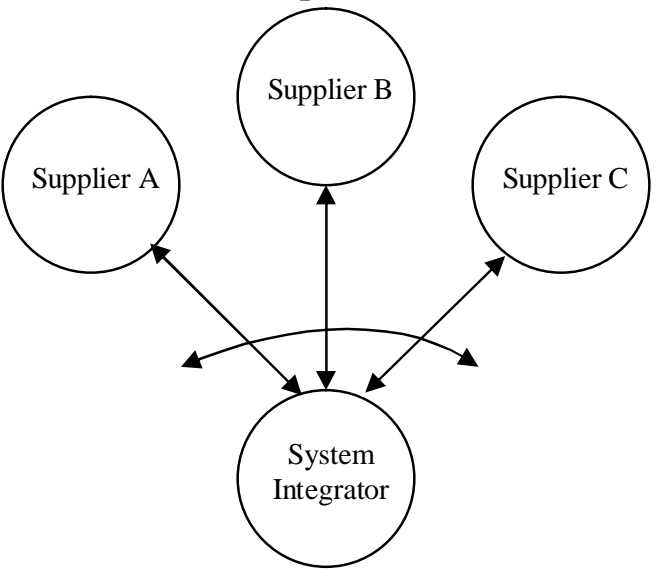
effectively on changes within the market. Within this structural, durable and joint relationship, improvement and changes emerge to increase the

\section{Figure 1 Concept of the EME}

overall performance and create a competitive advantage. The overall performance of the EME is the result of the interaction between and the integration of inter-company processes and therefore the improvement of performance should involve the generation, implementation and evaluation of improvement activities on intra-company level as well as the inter-company level (Cagliano 2000). Therefore the concept of continuous improvement should be applied to inter-company processes, transferring and extending the mechanisms, tools, practices and values of CI within the stand-alone company to the level of the EME.

\section{$\underline{\text { Continuous Improvement and Collaborative Improvement }}$}

Organisational change and especially improvements get much attention in the literature as well as in practice. Often in the literature, a distinction is made between major (radical) and minor (incremental) changes. Incremental improvement is a well-known concept and is widely discussed at the level of single firms by the literature on CI (Imai 1986, Bessant and Caffyn 1997, Boer et al. 2000). The concept of Continuous Improvement (CI) was developed as a new field in Operations and Innovation Management in relation to the Japanese practices of Kaizen. Many authors have contributed to literature of CI, such as Imai 1986, Deming 1986, Bessant and Caffyn 1997, Boer et al. 2000).

$\mathrm{CI}$ is defined as "the planned, organised and systematic process of ongoing, incremental and company-wide change of existing practices aimed at improving company performance" (Boer et al. 2000: 1). The problem with CI is that such a, at first sight very simple and attractive concept appears to be difficult to design, implement and develop successfully. "Despite its attractions, evidence suggest that CI often fails, or fails to take root in organisations which try to implement it. Arguably this is a problem of design and management of CI systems" (Bessant 1998). However, evidence provided by the 
(Euro)CINet has shown that among the major benefits of CI are increased business performance and 'people performance' (Boer et al. 2000).

But a strong limitation of the literature of CI is the unit of analysis, namely the single company. As competition is moving to the level of EME, continuous improvement cannot be confined anymore to the intra-company level. However, there is still a substantial lack of empirically grounded contributions and theories on the concept of CI in an interorganizational setting. CI can hardly be applied in inter-organisational settings due to organisational, geographical and time related barriers, and, therefore, need to be transferred and extended to the level of collaborative continuous improvement, leading to the concept of collaborative improvement. This is the specific focus of this paper.

Table 1: Commonality/difference between CI and CoI

\begin{tabular}{|c|c|c|}
\hline Area & Key components of CI & Additional key components to CoI \\
\hline Strategy & $\begin{array}{ll}\text { - } & \text { Clear strategic framework for CI } \\
\text { - } & \text { Long-term goals and short-term } \\
\text { targets } \\
\text { - Communication of CI strategy to all } \\
\text { employees } \\
\text { - Top management commitment } \\
\text { Long-term, company wide } \\
\text { perspective }\end{array}$ & $\begin{array}{l}\text { - Shared goals and vision with regard } \\
\text { to CoI } \\
\text { - Mutual understanding of CoI- } \\
\text { strategy of all the companies } \\
\text { - Company/EME commitment } \\
\text { towards CoI } \\
\text { - Long-term optimisation instead of } \\
\text { short-term orientation }\end{array}$ \\
\hline Culture & $\begin{array}{l}\text { - } \begin{array}{l}\text { Shared belief in the value of small } \\
\text { improvements } \\
\text { - } \\
\text { Belief that all employees have } \\
\text { creative potential } \\
\text { - Treating failure as a learning } \\
\text { opportunity }\end{array}\end{array}$ & 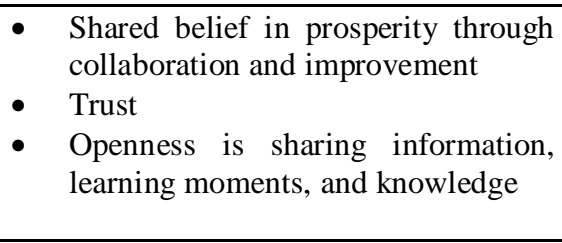 \\
\hline Infrastructure & $\begin{array}{ll}\text { - } & \text { Flattened hierarchy } \\
\text { - } & \text { Teamworking and flexibility } \\
\text { - } & \text { Devolution of decision making and } \\
\text { empowerment } \\
\text { - } & \text { Effective communication channels } \\
\text { - } & \text { Commitment to training and } \\
\text { - } & \text { CI facilitators } \\
\text { - } & \text { CI 'vehicles' such as problem solving } \\
\text { groups or CI teams }\end{array}$ & $\begin{array}{l}\text { - Effective communication channels } \\
\text { - CI 'vehicles' such as problem } \\
\text { solving groups or CI teams } \\
\text { - Devolution of decision making } \\
\text { - Commitment to exploiting and } \\
\text { exploring improvement potential } \\
\text { inside collaborative relationships }\end{array}$ \\
\hline Process & $\begin{array}{ll}\text { - } & \text { Formal CI/problem solving cycle } \\
\text { - } & \text { Capture and transfer of learning } \\
\text { - } & \text { Recognition and reward of CI activity }\end{array}$ & $\begin{array}{l}\text { - Capture and transfer of learning } \\
\text { between and within companies } \\
\text { - } \quad \text { Benefit sharing }\end{array}$ \\
\hline Tools & $\begin{array}{l}\text { - Company 'toolbox' with a range of } \\
\text { CI tools } \\
\text { - 'Toolbox manager' }\end{array}$ & $\begin{array}{l}\text { - } \text { EME 'toolbox' with a range of CoI } \\
\text { tools that are applied similarly } \\
\text { within the EME companies }\end{array}$ \\
\hline
\end{tabular}

In this paper Collaborative Improvement (CoI) is defined as "a purposeful inter-company interactive process that focuses on continuous incremental innovation aimed at enhancing the EME overall performance". It is simultaneously concerned with bringing about change in the EMEs, developing EMEs capabilities, and generating actionable knowledge. Finally, it is an evolving systematic change process that is undertaken in a spirit of collaboration and learning. Commonalities and differences between the CI and CoI are depicted in Table 1. 
Based on the definition for CoI, this paper will discuss the development of a model to support the analysis and redesign of how collaborative improvement is fostered and sustained within the EME.

\section{Research problem and methodology}

In order to implement collaborative improvement it is of vital importance to understand and highlight the needs and requirements of EMEs on how to organise, manage and support collaborative improvement activities. Therefore, we need empirical evidence to understand the needs and requirements with regard to organisational, managerial and technological tools. On the basis of the identified user requirements in a Dutch EME we will propose a preliminary Business Model of collaborative improvement, explaining how collaborative improvement can be sustained and supported in an EME context.

The research problem thus is:

What requirements can be identified for an EME to implement, support and sustain collaborative improvement activities within the EME?

The following research questions are formulated:

1. Which user requirements of an EME, in terms of organisational and managerial functionalities and mechanisms aimed at supporting the collaborative improvement process, can be identified?

2. Which barriers hamper the implementation and sustainability of collaborative improvement?

3. How can collaborative improvement in an EME context be modelled?

Empirical research was carried out to understand and highlight the need for collaborative improvement of the EME. The basis of the investigation of the user requirements is the current situation of collaboration within the EME.

Given the exploratory nature of this research, a case study methodology (Yin, 1984) was selected. An in-depth case study was carried out in a Dutch EME, consisting of the network of the system integrator Power Packer Europe BV and three of their suppliers. Interviews were conducted using an Investigation Framework, which was developed by the COIMPROVE consortium, including a questionnaire and an analysis protocol. The first main area addressed by the interviews analysed general information about the system integrator, the selected suppliers of the system integrator, the scope and goal of collaboration and primary processes between the firms within the EME. The second area explored the general overview of the collaborative improvement and seeks for specific and concrete examples of collaborative improvement activities performed in the past. The last area addressed the user requirements in terms of managerial and organisational needs and user requirements in terms of software support. The interviews with the system integrator were performed with employees from different functions, namely procurement, quality, production and logistics. Subsequently each supplier was interviewed at his own site, to catch his own opinion on the relationship with the system integrator. From the suppliers' site, interviews were performed with people from sales and engineering. The focus of the analysis was the relationship between the companies, trying to understand its evolution over time and the attempts to collaborate for improving the performance of the EME as a whole. The results of the in- 
depth case study were fed back to the EME in a joint workshop in order to consolidate the findings.

\section{The case study of the EME Power Packer Europe BV (NL)}

This section reports on the in-depth case study of how (collaborative) improvement currently takes place and how it could/should be organised within the EME. First the system integrator is introduced, followed by its suppliers that were included in the case study. The focus is on the relationship between the companies within the EME, their current collaborative practices, needs and areas of improvement.

\section{Power Packer Europe BV (NL)}

Power Packer Europe BV (PPE) is an independent subsidiary of the USA-based parent company, Actuant Corporation. PPE employs 425 staff with a turnover in 1999 of 100 million Euros. The turnover has tripled in 3 years. Power Packer Europe (PPE) is specialised in 'Motion Control' systems for the different markets of:

- automotive: electro-hydraulic actuant systems for operating soft tops or retractable hard tops on convertible cars as well as opening/closing car trunks,

- truck: hydraulic and electro-hydraulic cab tilt systems, cylinders for auxiliary steering systems and cylinders for boggle lift systems,

- marine: hydraulic and electro-hydraulic steering systems for pleasure boats, trim/tilt units for outboards, electro-hydraulic operating systems for hatches and masts,

- medical: systems for hydraulic height adjustment of beds, stretchers and tables, electrohydraulic systems for adjustment of scanner tables,

- agriculture market: cylinders and valve blocks for reversible ploughs, hydraulic nonstop systems and cylinders for adjusting mobile spray and sprinkler systems.

PPE sees itself in a niche market, dominantly automotive and truck. Within Europe there are only 2 main players in both markets, of which PPE is one. On a global scale there are a few more players. The competition is known, heavy and mainly on price. PPE observes a shift towards a commodity market. In this new market the order-winning criterion is price, whereas quality and technology are qualifiers. For a company in the automotive industry it is a main challenge to constantly monitor the cost-structure in order to remain profitable. This is a result of the price pressure from the OEMs, the increase in price of raw materials and contracts on long-term delivery schedules.

PPE has as a strategic objective to product zero-defect products against the lowest total cost of world-class suppliers to satisfy PPE requirements on quality, cost and delivery. To realise this strategic objective PPE selects suppliers that: (1) apply for continuous improvement; (2) are able to realise early supplier involvement (ESI) starting from the first conceptual phase to guarantee a maximum use of the supplier's knowledge, which increases efficiency and reduces time to market and cost; (3) that comply with world class standards. The supplier base of PPE is international, ranging from small local companies to more world-level players, and continuous improvement and continuous cost reduction are an integrated part of PPEs policy. Continuous benchmarking is used to compare suppliers against the best in class. Cost analyses with suppliers are based on open book calculations in order to achieve targets. PPE aims for close co-operation and long-term agreements with a limited number of suppliers. 


\section{Suppliers}

The suppliers selected by PPE to be involved in the research project represent different types of relationships with PPE. The relationship is assumed to be determined by both structural characteristics of the company, such as size, location, competence, dependence of each other, and the object of the interaction that could be the supply of finished parts or just the outsourcing of some activities. Figure 2 gives an overview of the PPE EME and the main parts that are delivered by the suppliers to PPE.

\section{SchmitterSysCo $\mathrm{GmbH}$}

SchmitterSysCo is a medium-sized company that is specialised in the production and development of cylinder-tubes for the automotive industry. The firm originally started in 1968, but after some mergers SchmitterSysCo have become part of the Schmitter-Gruppe. The company has 160 employees, achieving a turnover of 22 million EURO in 2000. The relationship between SchmitterSysCo and PPE is fairly young and therefore chosen by PPE to be included in this research project. PPE has started to be supplied by SchmitterSysCo because the company is able to handle the entire process from buying the raw material to delivery of cylinder-tubes, regarding the fact that PPE buys the raw material for the current supplier. In this way PPE hands over the purchasing activity, which allows PPE to focus on its own core-activities. Further, SchmitterSysCo is seen as a real "automotive supplier" on A-level, which is an important aspect since the automotive industry is known for its specific characteristics. Since the relationship is young, currently there is no track record of CoI activities between the companies. Both, however, are very interested in long term, structural CoI.

\section{MEVO Precision Technology}

MEVO Precision Technology is a small/medium-sized company, which is specialised in the production and delivery of fine mechanical parts for the high-tech industry. Its products are supplied to customers in the automotive, agriculture, optical, medical and measurement/ control industry. The company has 55 employees and has a subsidiary, MEVO Sro, in Slovakia, with 35 employees, supplying the Dutch company. MEVO supplies PPE with parts for the pump for opening the roof and tilting the cabin of a truck (i.e. rotor). The relationship resembles that of a traditional supplier-customer relationship, where problems in production are the inducement to start an improvement project. Faults and problems trigger the need for improvement and therefore CI is much more reactive than proactive. This company was selected to be involved in the research project because of its long-term relationship with PPE and its collaboration on improvement projects. The intention of both the companies is to increase collaboration and work towards early supplier involvement.

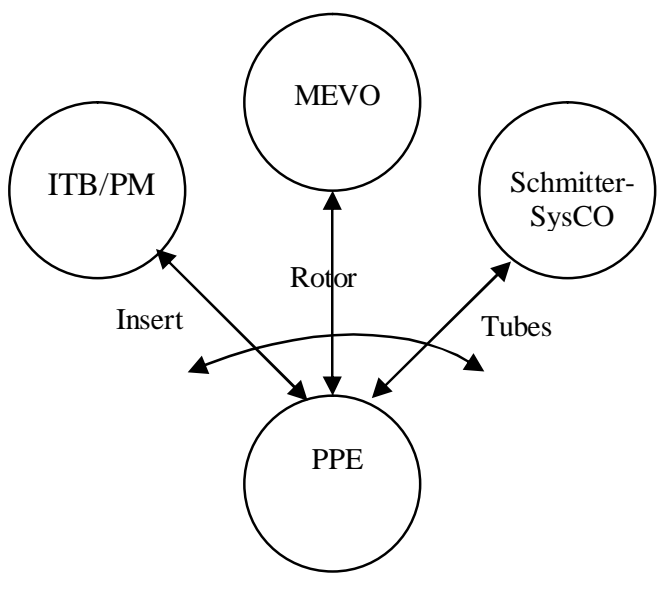

Figure 2: PPE EME 


\section{$\underline{\text { ITB/PM BV }}$}

ITB/PM BV is a part of the ITB group, which has approximately 200 employees. ITB/PM is specialised in the design and production of automotive, medical, pharmaceutical plastic precision parts and assembled products. ITB/PM supplies PPE with plastic moulding products (i.e. insert). There is a close relationship between the companies. ITB/PM is familiar with the processes of PPE, requirements and wishes with regard to products. The relationship includes some elements of early supplier involvement. The trigger for CoI activities is balanced between the two companies, and organised through improvement projects. During CoI activities an extensive face-to-face contact between different people and different functions is used for exchanging and sharing information. This company was selected because of its intensive collaboration with PPE over a number of years.

\section{The requirements and areas of application for Collaborative Improvement}

The case study within the PPE EME was performed to understand and highlight the areas of application of collaborative improvement and the requirements of the companies with regard to organisational and technological functionalities and mechanisms of collaborative improvement. The results of the investigation are discussed in the following subsections.

\section{Areas of application}

The areas of implementation of CoI are on the level of "collaborative operations", that is, day-to-day business at inter-company level in which improvement needs emerge. As far as collaborative operations are considered, the following investigation of user requirements were identified:

- goal sharing and mutual understanding,

- order management,

- quality management,

- manufacturing,

- change order management.

These five areas of implementation of CoI highlight two different levels of opportunities for CoI. The fist level concerns collaboration on an operational level, where performance is measured in terms of time, quality and cost according to the assessment of PPE of its suppliers. The building blocks of collaborative operations are the work practices, i.e., the set of tools, techniques and organisational/managerial arrangements used to perform the day-by-day work. The second level refers to a number of processes (mutual knowledge and goal sharing) that are concerned with relationship management. The processes of relationship management are strategic, long-term activities oriented at the development and management of inter-company relations that support operational practices. The case study highlighted and stressed the need of suppliers to be involved in a very early stage in the NPD process of PPE, the so-called early supplier involvement (ESI).

The interdependency between the five identified areas is shown in figure 3 . 
Mutual knowledge/goal sharing

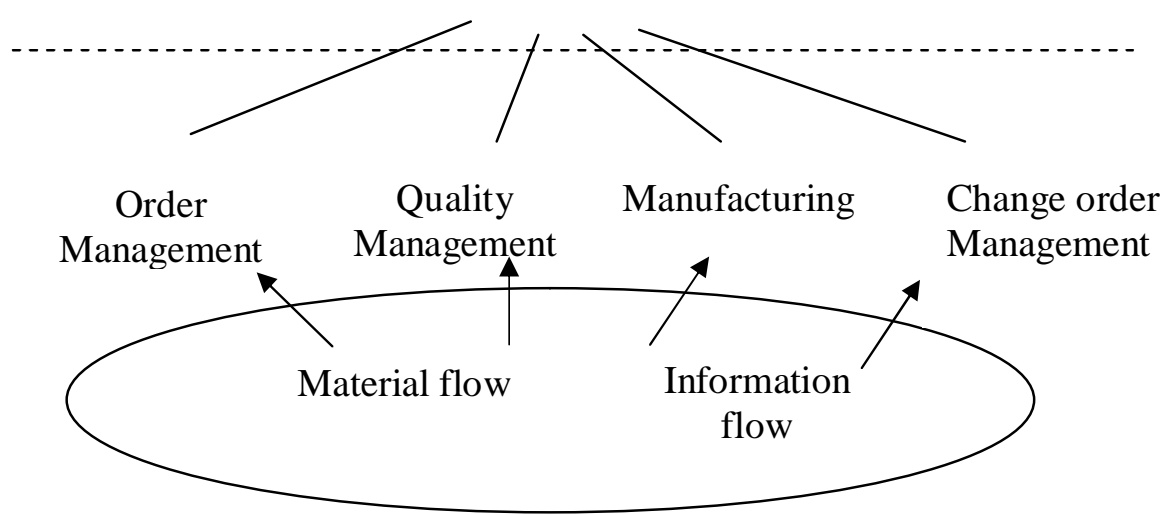

\section{Figure 3 The five improvement areas of collaborative operations}

First, order management refers to the fulfilment of orders that are performed across the boundaries of the companies within the EME. The improvement of performance in this area requires the integration of all activities needed to fulfil the orders and collaboration through a rich and timely information exchange on order status, demand forecast and administrative issues. For example, MEVO can access all the information needed for the fulfilment of an order. However, information exchange still depends heavily on face-to-face contact and MEVO asking for the information. Tools and mechanisms supporting a more formal and structured way of exchanging information in the area of order management can give great advantage to the companies within the EME.

The second area for Collaborative Improvement is quality management. Requirements towards quality within the automotive industry are nowadays extremely high, not only with regard to the product and process, but the entire organisation and supply chain are nowadays big issues within quality management. Therefore, the whole scope of methods, tools and techniques will be referred to in this area. An example of the use of different methods and techniques within quality management can be found in the relationship between PPE and MEVO. It was revealed that during assembly an inner circle of a rotor became eccentric. To solve this problem a project team was installed that searched for the cause of the problem. The team worked according to a problem solving technique: causes were clustered, solutions were identified and clustered and people started working on the most important problems.

Third, the opportunities to use $\mathrm{CoI}$ in the manufacturing process refer to the possibility of improving products and processes, both for cost reduction and quality improvement. An example of product improvement and cost reduction can be found in the case of PPE and ITB/PM. This company redesigned a gearwheel in order to reduce parts, costs and assembly time, simultaneously. The final design and final product did meet requirements and functionalities and still was cheaper compared to the former gearwheel. PPE was able to lower the cost while enhancing the required quality.

The final area that can provide opportunities for $\mathrm{CoI}$ is change order management. A timely exchange of relevant information on design changes and related order updates could greatly 
improve the effectiveness and efficiency of operations, especially when changes concern a complex supply network.

\section{Enablers and Disenablers for Collaborative Improvement}

Based on the case study requirements the needs of the companies within the EME toward Collaborative Improvement were identified. First, we discuss some general enabling factors for Collaborative Improvement. Second, we discuss the organisational, managerial and technological needs of the companies, and this section ends with some barriers to implementation of Collaborative Improvement activities within the EME.

\section{Enablers for successful Collaborative Improvement}

Enabling factors for successful CoI on an EME-level were identified as:

1. Goal sharing along the supply chain. This factor is required in order to allow actual collaboration and to finalise efforts to effective results, with benefits perceived by all the actors. Potential improvements don't take place because of misalignment of objectives and priorities between customer and supplier.

2. Trust and long-term perspectives. Although the CO-IMPROVE project is deliberately focussed on EMEs that pursue prosperity through collaboration, consensus and synergy, at the end of the day there is always partner interest in terms of burdens and goods that have to be shared and agreed upon. The business model needs to consider how this political process happens. Trust and long-term perspectives play an important role in this process. The contemporary agreements may not be balanced with respect to short term benefits for the partners, but for the long term they should be.

3. Organisational improvement and ICT support. The third consideration, derived from the analysis of the results of the interviews, is that both organisational improvement and ICT support are needed. The former is required to enable the exploitation of the improvement potential hidden inside collaborative relationships, while the latter both increases this potential and enables activities that otherwise would be very difficult, such as distance interaction and knowledge management.

4. Openness. Not only openness in sharing information to the suppliers is required, but also readiness to discuss problems and faults with each other in order to generate greater benefits for the entire EME. Although the openness is restricted by characteristics of the automotive market the EME is in, it is still essential for the collaborative improvement process.

\section{Disenablers to Implementation}

Companies must be aware of these barriers, since they will affect the implementation and sustainment of collaborative improvement processes. The barriers identified mainly concern the relation between the companies, e.g. culture, lack of trust or different interest and politics. Also, some barriers are related to network specific characteristics and lack of resources:

- Diffusion of techniques that are used and applied: PPE uses quality and logistics techniques and methods (8-D technique and Failure Mode and Effect Analysis), which are not used and applied by its supplier(s), and also the other way around,

- Short-term orientation instead of a long-term optimisation,

- Mental map of companies: The legacy of old attitudes in companies, where people can have problems in understanding why they should collaborate with companies,

- In-depth knowledge of processes and organisation on the part of the supplier, 
- Lack of trust: There is a limited level of openness within the automotive industry and therefore companies are not willing to share much information with suppliers, partners and customers,

- Different interests and politics on the side of the suppliers: Different suppliers within the supply chain have different interests, and as such will focus on different issues,

- Communication: Problems of suppliers are not always reported fully and therefore additional meetings are necessary to find the causes of the problem. Or the problems are just reported and documented and nothing is done,

- Certification: Due to very strict quality requirements, which are characteristic of the automotive industry, there is a need for certification of product, process and the company. This can limit the possible changes in product and process, and therefore Collaborative Improvement,

- Lack of knowledge: Collaborative Improvement can be hindered by personal turnover which can have consequences for the alignment within projects. New people may have a lack of knowledge and are not familiar with the way of working,

- Lack of priorities: Problem-solving projects are based on solving the main problems, leaving several smaller problems/issues unsolved. Once the main problem is solved it is back to usual business

The findings from the case study indicate that improvement activities frequently have the character of (ad-hove problem driven) improvement projects, rather than that of collaborative, structural and pro-active improvement processes. The activities are centred around product and process problems and are driven by the supplier-assessment of PPE with regard to issues involving quality, cost and delivery. In this way the initiative to start with improvement lies with PPE. In general it can be observed that the improvement activities merely develop on the dyadic relationships between PPE and individual supplier, trying to improve performance on quality, cost and delivery of the two companies on the dyad. Improvement activities are not yet developed on an EME/collaborative level. Although the need for this kind of collaborative improvement relationship is recognised by the companies and actively striven for, the relationship has not yet matured to this level.

A workshop was organised to discuss the results of the requirements with PPE and its suppliers. Also other interested companies were invited to this workshop in order to "check" the requirements and disseminate findings. This workshop teased out different opinions and definitions surrounding collaborative improvement. By comparing the PPE EME's experiences with those of other companies it was possible to further mitigate subjectivity of the research and add to the generalisability of the findings.

The Dutch results were also presented within a CO-IMPROVE consortium meeting and compared to the Danish and Italian EME results.

\section{Collaborative improvement model}

The development of the business model for CoI has started with the definition of collaborative improvement, as an inter-company process aimed at improving the EME performance. Through the enhancement of practices used for managing inter-company processes, the collaborative work practices are improved and the relationship between the partners is strengthened. Collaborative operations become more effective, thus improving the performance of the EME as a whole. At the same time, by performing collaborative improvement activities, the EME creates capabilities based on existing behaviours, thus 
providing competitive advantage to the EME. These capabilities in turn can enhance the evolution of collaborative improvement. This results in continuous improvement of capabilities of the individual firms within the EME and the parent EME itself.

In summary, there are three logical levels that can be distinguished (see figure 4):

- Collaborative operations;

- Collaborative improvement processes, and;

- Collaborative capability building.

This research has emphasised the collaborative improvement process level and its mutual relationship with the two other levels.

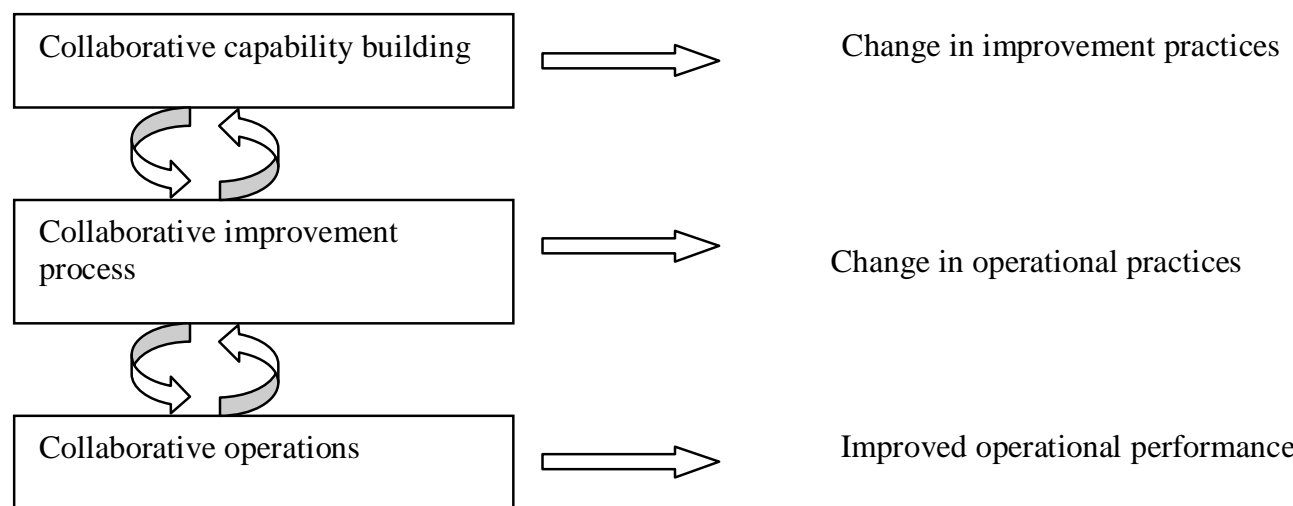

Figure 4 Framework of collaborative improvement

On the lowest level, collaborative operations (i.e. manufacturing, quality management, order cycle management and change order management), identified and discussed in a previous section of this paper define the processes concerned with the collaborative operations and performance in terms of quality, time, flexibility and cost at the EME level. At the middle level, the activities within the collaborative improvement process are: goal sharing and alignment, collaborative improvement generation, collaborative improvement implementation and collaborative improvement evaluation. Goal sharing is one of the general identified enabling factors, and is required to allow actual and effective collaboration and potential improvements to take place through the alignment and definition of shared objectives and priorities. At the highest level, collaborative capability building refers to the ability of the companies within the EME to consolidate improvement processes, values and behaviours in capabilities to accelerate the process of building and applying new improvement activities.

The case study has indicated that dyadic improvement relationships can be identified. These relationships are mainly on the level of company-based improvement and/or cooperative improvements. The case study highlights the fact that within the PPE EME there are no common goals or strategy towards collaborative improvement. Although the companies state that collaborative improvement is recognised and planned for, the relationships have not yet developed to this extend. The improvement projects that have been identified were problem-driven in the sense that operational problems at the site of PPE are the inducement for starting an improvement project. 


\section{Conclusions and Future Research}

Continuous improvement is a consolidated concept in managerial theory and practice and is considered vital in today's business environments. But a strong limitation of the literature of $\mathrm{CI}$ is the unit of analysis, namely the single company. As competition is changing, we need to transfer and apply the concept and the practical elements of CI to the interorganisational setting. However, there is still a substantial lack of empirically grounded contributions and theories on the concept of $\mathrm{CI}$ in an inter-organizational setting. Within this paper we have provided empirical evidence and build on existing concepts of CI in order identify the areas where continuous improvement in an EME context is and could be applied.

The empirical evidence presented in this paper supports the development of a model of CoI that can be considered a general framework for the collaborative improvement process. The model distinguishes between collaborative operations, collaborative improvement and collaborative capability building and the dynamic relationship between the three levels.

Extending and transferring the concept of CI to an inter-organisational setting implies to research factors that affect the development of the process of collaborative improvement, such as trust, shared goals and vision, strategy, and political behaviour. Although these factors have an affect in itself, the configuration and interaction of the factors is important to research.

Many issues of collaborative improvement need to be addressed and require more detailed analysis and development in future research:

1. The impact of enablers and disenablers on the process of collaborative improvement,

2. Detailed analysis of the characteristics of inter-organisational CI in comparison to the concept of CI in the context of the stand-alone company,

3. The implementation process of collaborative improvement,

4. Detailed analysis of intercultural differences in the approaches towards collaborative improvement in Denmark, Italy and the Netherlands.

Future research in the CO-IMPROVE project will address these topics through a two year action research approach in order to build theory on collaborative improvement and to identify fields of application and the benefits of collaborative improvement in practice.

\section{References}

BESSANT, J., CAFFYN, S., 1997, High-involvement Innovation through Continuous Improvement, International Journal of Technology Management, 14, (1), 7-28.

BESSANT, J., 1998, Developing Continuous Improvement Capability, International Journal of Innovation Management, 2, 409-429

BOER, H., BERGER, A., CHAPMAN, R., and GERTSEN, F. 2000, CI changes: from suggestion box to organisational learning, Continuous Improvement in Europe and Australia, Ashgate Publishing Ltd., Aldershot

BUSBY and FAN, 1993, The extended manufacturing enterprise: its nature and its needs, International journal of technology management, 8, (3,4,5), 294-308

CAGLIANO, R., 2000, Integration Mechanisms of Inter-company Processes, Proceedings of the $1^{\text {st }}$ world conference of POM

COASE, R.H., 1937, The nature of the firm, Economica N.S.

DEMING, W.E., 1986, Out of the Crisis, MIT Press, Cambridge, MA.

DOUMA, M.U., 1997, Strategic Alliances, Fit or Failure, Drukkerij Elinkwijk BV, Utrecht 
DYER, J.H., 1997, Effective Interfirm Collaboration: how firms minimize transaction costs and maximize transaction value, Strategic Management Journal, 18, 535-556

HAMEL, G., DOZ, Y. AND PRAHALAD, C.K., 1989, Collaborate with your competitors - and win, Harvard Business Review, January-February

HARLAND, C.M., LAMMING, R.C., and COUSINS, P.D., 1999, Developing the concept of supply strategy, International Journal of Operations \& Production Management, 19, (7), 650-673

IMAI, M. (1986), Kaizen. The key to Japan's Competitive Success, McGraw-Hill Publishing Company, New York

KANTER, R.M., 1994, What thinking globally really means, Harvard Business Review, Global Strategies, Harvard Business School Press

LAMMING, R. 1993, Beyond partnership, Pentice Hall, London

MOHR, J., and SPEKMAN, R., 1994, Characteristics of Partnership Succes: Partnership Attributes, Communication Behaviour, and Conflict Resolution Techniques, Strategic Management Journal, 15, (2), 135-152

PORTER, M.E., 1987, Managing value - from competitive advantage to corporate strategy, Harvard Business Review, May-June

RICH, N., and HINES, P., 1997, Supply-chain management and time-based competition: the role of the supplier association, International Journal of Physical Distrubution \& Logistics Management, 27, (3/4), 210-225

RING, P.S., and VAN DE VEN, A.H., 1992, Structuring cooperative relationships between companies, Strategic Management Journal, 13, (7), 483-498

SMITH, H.L., DICKSON, K., SMITH, S.L., 1991, There are two sides to every story: Innovation and collaboration within networks of large and small firms, Research Policy, 20, 457-469

SPEKMAN, R.E., KAMUAFF JR, J.W., 1998, An empirical investigation into supply chain management, A perspective on partnerships, International Journal of Physical and Logistics Management, 28, 8

STOCK, G.N., GREIS, N.P., KASARDA, J.D., 2000, Enterprise logistics and supply chain structure: the role of fit, Journal of Operations Management, 18, (5), 531-547

WILLIAMSON, O.E., 1983, Credible commitments: using hostage to support exchange, American Economics Review, $\mathbf{7 5}$

YIN, R.K. 1994, Case study Research, Design and Methods, $2^{\text {nd }}$ edition, Sage Publications Inc., London 